\title{
Bandwidth-aware Energy Efficient Flow Scheduling with SDN in Data Center Networks
}

\author{
Guan $\mathrm{Xu}^{\mathrm{a}}$, Bin Dai ${ }^{\mathrm{a}}$, Benxiong Huang ${ }^{\mathrm{a}}$, Jun Yang ${ }^{\mathrm{a}}$, Sheng Wen ${ }^{\mathrm{b}}$ \\ ${ }^{a}$ School of Electronic Information and Communications \\ Huazhong University of Science and Technology \\ Wuhan, China \\ ${ }^{b}$ School of Information Technology \\ Deakin University \\ Melbourne, Australia, VIC 3125
}

\begin{abstract}
Nowadays the energy consumption has become one of the most urgent issues for Data center networks. For general network devices, the power is constant and independent from the actual transfer rate. Therefore the network devices are energy efficient when they are in full workload. The flow scheduling methods based on the exclusive routing can reduce the network energy consumption, as the exclusive routing paths can fully utilize all their links. However, these methods will no longer guarantee the energy efficiency of switches, as they handle flows in priority order by greedily choosing the path of available links instantaneously. In a previous work we proposed an extreme case of flow scheduling based on both link and switch utilization. Herein we consider general scenarios in data center networks and propose a novel energy efficient flow scheduling and routing algorithm in SDN. This method minimizes the overall energy for data center traffic in time dimension, and increases the utilization of switches and meet the flow requirements such as deadline. We did a series of simulation studies in the INET framework of OMNet++. The experiment results show that our algorithm can reduce the overall energy with respect to the traffic volume and reduce the flow completion time on average.
\end{abstract}

Keywords: Energy Efficiency, SDN, DCN, Exclusive Routing

\section{Introduction}

Cloud is a "hot" research topic at the moment, with interests in security [1-6], privacy [6, 7], forensics [8-15], and achieving efficiency [16] in Data Center Networks(DCNs). For D$\mathrm{CNs}$, the increasing energy consumption has been restricting the sustainable growth of cloud services and raises economic and environmental crisis. The Power Usage Effectiveness (PUE) of typical DCNs ranges from 1.3 to 3.0, as reported from the Green Grid in 2012 [17]. Among data center energy consumptions, the increasingly salient network energy contributes $20 \%$ of the whole power consumption typically [18] and even $50 \%$ when we use the server power management techniques $[19,20]$. Basically, the DCN architectures built with rich connections can achieve high network performance by multi-party routings, however, the architectures waste energy for two reasons. Firstly, the network devices are in low utilization as the DCN traffic is typically smaller than the peak, especially in the traffic valley time [21]. On the other hand, the power of network devices is nearly constant when the traffic speed changes [22].

Traffic aggregation and scheduling techniques are widely adopted in the existing work of energy efficient DCN networking. The former can shape the traffic distributions while the latter can manage the traffic admissions. The traffic aggregation methods [23-26] can aggregate traffic into fewer switches/ports

Email address: nease.dai@gmail.com (Bin Dai) and turn more switches/ports into sleep to obtain a minimum power for a given traffic matrix. The traffic scheduling methods can suspend the delay-insensitive traffic until energy efficient paths are available, achieving more energy savings with acceptable completion time. As an extreme case of the traffic scheduling, the exclusive routings [27, 28] keep the rule that each link serve a flow at a time. In this case, all active links can be fully utilized for network constrained traffic, such as the block transfer in a shuffle phase of a MapReduce application. The active links will be of the maximum energy efficiency. In an ideal energy efficient scenario, the DCN traffic should route with a minimum usage of switches and links.

The energy efficient flow scheduling can influence the network performances, such as network throughput and delay. For example the method exploits the Earliest Deadline First (EDF) policy and forwards flows with earlier deadline first [27]. The flow scheduling method in [28] exclusively forwards flows according to the flow priorities in terms of flow size or flow deadline. The exclusive routing can make full use of links as it allows flows monopolize the path and flows can be transmitted with full bandwidth of links. However, these flow scheduling methods are not energy efficient enough, as they consider energy efficiency flow scheduling in priority order of flow size or deadline directly. As the characters of DCN flows, such as flow size and deadlines, can be predicted by the typical DCN traffic patterns or queried from the DCN controller, it is possible to schedule flows with the deadline constraints instead of 
scheduling flows in deadline order. The flow scheduling with the deadline constraints can achieve more energy savings with affordable delays. Therefore, it is required to build an energy efficient problem with the requirement of deadline in DCN.

Software Defined Networking (SDN) [29] has been a revolutionary and promising network architecture to promote network management functions. The architecture is generally divided into SDN controllers and SDN supported switches. The SDN controller is a centralized network manager software, designed to instruct all SDN supported switches to forward network flows. On top of that, the SDN controller provides applicationfacing northbound API to enable unprecedented programmability, automation and network control for enterprises and carriers. For example, PANE [30] is a network aware application framework designed for SDN that can allocate available bandwidth to applications, with the SDN controller monitoring network flows and link bandwidth resources. Even when there is no available bandwidth, PANE will calculate the completion time of existing flows and then inform new applications the available sending time. With the PANE framework scheduling the link bandwidth resources in the time dimension, one question to handle the energy saving issue is naturally asked: Can we apply this centralized flow schedule to energy efficient data center networks for high efficiency and agility in terms of energy consumption?

In this paper, we first introduce the energy efficiency problem for network-constrained network flows with deadline in D$\mathrm{CN}$. The DCN servers generating network-constrained network flows faster than the transmission capacity of the network. And then we propose a novel Energy Efficient network management method by exploiting the flow scheduling and exclusive routing with constraints of flow deadlines. The design is based on the observations that DCN flows with exclusive routings can be consolidated in time dimension. In our energy routing algorith$\mathrm{m}$, flows have to wait for the approval of sending from the SDN controller. To evaluate the performance, we implement our algorithm in simulation platform with typical DCN topologies. Specifically, this paper has the following contributes:

- We describe the energy efficiency problem of the networkconstrained network flows with the requirement of deadline in DCN and model this problem as a linear programming;

- We propose a bandwidth-aware energy efficient routing algorithm with SDN to solve the problem;

- Through a series of simulation studies in OMNeT++ platform, we evaluate our algorithm and show that our algorithm can achieve higher energy efficiency and lower completion time in Fat-Tree and BCube topologies.

In the following sections, we state the problem of energy efficient network for DCN in section 2, overview the related work in section 3 , and then offer the energy model in section 4 with the flow scheduling algorithm designed in section 6. Evaluations with simulations are provided in section 7 .

\section{Problem Statement}

\subsection{Network Energy Problem}

The network energy saving problems in DCN is first stated in [20]. For typical DCNs, the networks are generally designed for the traffic peak in DCN rather than the real-time traffic [21]. However, the commonly used routing algorithms such as ECM$\mathrm{P}$ [16] are designed for load balance, and cannot make full use of the network energy consumption, as the power of the switches are not speed-scalable [22]. Therefore, an active switch with low or high traffic will consume the same energy.

\subsection{General solutions}

To decrease the network energy consumption in DCN, existing work is on how to maximize the energy efficiency of network elements(NEs). With flow consolidation operations, the DCN flows can be directed to a sub-topology with the minimum links and switches, leaving other NEs in sleep or shutdown mode to save energy. The sub-topology will sacrifice the network performance, and bring larger network delay and unreliable links with higher utilization. Therefore, an ideal energy efficient flow consolidation method is to balance the energy saving and the traffic requirements at the same time.

As the first work on flow consolidation for energy savings, ElasticTree [25] is designed to find a minimum sub-topology for a given traffic matrix. Since that a fixed traffic matrix is impractical for dynamical traffic in DCN, the correlation based flow consolidation [31] considered the time variable character of the DCN flows. They consider the correlation of DCN traffic and consolidate flows with low correlation, as not all flows peak at the same time.

The flow consolidation methods hardly guarantee that al1 links are fully utilized from the perspective of link utilization. When the sub-topology serves the consolidated flows, not all links are serving flows with the same volume of the link capacity. This motivates a potential method for extra savings. For a given set of flows on the same link, the overall completion time will be the same no matter how much bandwidth are assigned to each flow. Therefore, as an extreme case of flow scheduling, the exclusive routing guarantees that each flow will exclusively use its path links, leading to the achievement of $100 \%$ link utilization. Based on the exclusive routing, flow scheduling methods have been proposed in $[19,32]$.

\subsection{Unsolved cases}

For the flow scheduling methods with exclusive routing, flows can preemptively route along the links of other flows with lower priorities. In [28], switches are chosen by a greedy method that can be implemented easily. However, the method cannot globally optimize the energy consumption. An example will be presented in section 6 that the greedy method of switches selection can be improved potentially. 


\section{Related Work}

In the literature, there are energy efficient optimizations for DCN servers. Ding et al. [33]considered the energy consumption savings of physical servers in cloud, where the typical servers that are not designed with energy saving technologies of hardware, such as dynamic voltage and frequency scaling (DVFS). They defined the performance-power ratio for heterogeneous physical servers, and proposed a virtual machine (VM) placement method to consolidate VMs to physical servers with higher performance-power ratio.

As the hardware processors can be designed to support DVF$\mathrm{S}$, there are energy efficiency optimizations on dynamic voltage scheduling and multi-core CPUs [34-36]. Wu et al. modeled the dynamic power as a convex function of the processor speed, and proposed an blocking-aware method [34] to dynamically schedule real-time tasks and adjust the processor speed. Pal et al. proposed a dynamic core allocation method [35] for video decoding applications at runtime, which can consolidate decoding tasks on fewer cores and shut off unused cores to save energy. They observed that using more cores brings shorter decode time while using fewer cores is more energy efficient, as the switching operations between cores will consume extra energy. Carabas et al. minimizing the energy consumption of DC$\mathrm{N}$ servers, by maintaining the optimal mapping between VMs and physical servers, and migrating VMs according to the mapping [36]. They follow the energy model that the energy consumption of servers increases more than linear when the server load is increased. Therefore, their mapping will not consolidate workload into a minimal set of servers. Jiang et al. [37] considered the energy consumption of GPUs, and proposed an algorithm to optimize the VM selection and allocation policies based on the utilization of both CPU and GPU.

Besides the energy efficient methods for servers, network energy saving are also studied in recent years, which can be divided into flow consolidation [26] and scheduling [28] methods. Generally, flows will share link bandwidth by fair sharing routings (FSR) according to protocols such as TCP. At the same time, the flow rate depends on its bottleneck link. The flow consolidation methods are designed to increase the utilization of active NEs by aggregating flows into a minimum set of links. The flow scheduling methods can further determine the sending order of flows.

The flows consolidation can be implemented in a greedy way. For example, the method in [26] sorts flows incrementally by the average flow rates and then find and reserve routings for flows with FSR from the highest rate flow to the lowest. In this way, the allocated flows will share the links fairly with their link bandwidth pre-reserved. However, the flow rate allocation is not precise, and the deployed flow rate will be larger than the average value as required for reliability, which will decrease link utilization. In the implementation of the flow consolidation, the order of flows can be designed with the constraints of the flow deadline. As an extreme case of flow reserving, exclusive routing (EXR) has been exploited to guarantee fullyutilized links without breaking the rule of fair sharing [28]. When flows are of time-varying rate, the flow consolidation methods consider the flow correlation [27, 31], where flows can be consolidated as the DCN traffic flows never peak at the same time.

However, the flow consolidation methods leave some unshared links in low utilization, which motivates the innovation$s$ based on exclusive routing. The exclusive routing reserves links for one flow exclusively, so that flows can transfer in the speed of link bandwidth, which simplifies the bandwidth allocation for homogeneous DCN structures, such as FatTree or BCube. The homogeneous link capacity will guarantee that the exclusive routing can make full use of links along the path. The exclusive routing with preemptive scheduling [28] brings a simply way of energy efficient DCN. They consider the network performance of energy efficiency with the flow deadline and completion time as the secondary objective, and design the flow scheduling with the priorities that can be flow deadline and flow size separately. For example, the flows with earlier deadline can be assigned a higher priority in routing for performance of flow deadline, while flows with smaller flow size can be assigned a higher priority for performance of flow completion time. The methods [28] and [26] are both incremental approaches. However, the utilization of switches is not guaranteed to be the minimal one from the overall perspective.

With flow size and link bandwidth available in SDN architecture, the ending time of a flow can be deterministically calculated so that we can schedule unallocated flows in queues to increase the flexibility of flow scheduling method. In the previous work [38], we proposed an energy efficient networking method that combines exclusive routing and flow scheduling in DCN.

In this work, we consider the energy consumption during the flow transfer time and extend the flow scheduling to time dimension. With heuristic method to search the solution of the flow groups that consumes the minimum energy, flows are scheduled and transferred with the active flow set and the suspended flow set.

\section{Model of Network Energy}

It is known that the energy consumption of a switch is not proportional to the traffic load [22] but to the dynamic part that constitutes the power consumption of a switch. For a working switch, the fixed energy consumption distributes on fans, chassis, switching fabric, etc., while the dynamic part usually refers to the working ports. A port consumes almost full power in an active state, but can be set to the sleep mode with nearly zero power consumption. For example, the switch Catalyst 6500 [39] has 2 linecards and 48 Gigabit ports in each linecard, with the power of the fixed part $472 \mathrm{~W}$ and that of each port $3 \mathrm{~W}$, the switch Nexus 2224 TP [40] has a linecard and 24 Gigabit ports, with the fixed power part $48 \mathrm{~W}$ and each port $2 \mathrm{~W}$. The total dynamic part consumption of $288 \mathrm{~W}$ will occupy $37.9 \%$ of the switchs power for Catalyst 6500, and for Neus 2224 TP the total dynamic part consumption of $48 \mathrm{~W}$ occupies $50 \%$ of the switchs power. Therefore, the working ports are required to be considered in designing energy efficient routings. 


\subsection{Problem of Network Energy}

Recommended by IEEE 802.3az [41], it is feasible to turn the idle port into sleep to save the network energy, due to the considerable power consumption to a switch from a dynamic part. The following equation is used to calculate the total amount of network energy consumed for transmitting traffic flows.

$$
E=\sum_{i \in W}\left(F_{i} \times T_{i}+\sum_{j \in P(i)} D_{i, j} \times T_{i, j}\right)
$$

which counts energy of each switch $i$, where $W$ denotes the switches in the network, $P(i)$ denotes the ports of the $i-t h$ switch, $F_{i}$ denotes the fixed power consumption in switch $i, T_{i}$ denotes the working time of switch $i, D_{i, j}$ denotes the dynamic power consumption of port $j$ in switch $i$, and $T_{i, j}$ denotes the working time of port $j$ in switch $i$.

As the newly proposed DCN structures such as FatTree and BCube are always built on homogeneous switches for scalability and efficiency [16]. We assume that the DCN is constructed by identical switches, each of which consumes fixed power $F$ and dynamic power $D$. Let $B$ denote the capacity of each port, Eq. (1) can be transferred into Eq. (2).

$$
E=F \times \sum_{i \in W} \frac{A_{i}}{B \times O_{i} \times|P(i)|}+D \times \sum_{i \in W} \sum_{j \in P(i)} \frac{A_{i, j}}{B \times O_{i j}}
$$

where $A_{i}$ and $A_{i, j}$ denote the aggregate traffic amount traversing switch $i$ and its port $j, O_{i}$ and $O_{i, j}$ denote the average occupation ratio of switch $i$ and its port $j$ in the working time, $|P(i)|$ denotes the number of ports for the $i-t h$ switch, respectively. Inspired by Eq.(2), the network energy consumption can be effectively reduced by increasing the occupation ratios of switches and ports, while turning idle ones into sleep mode. Therefore, a power-efficient network management approach is required to improve the occupation ratio of switches/ports to as high as possible.

\section{Bandwidth-aware Energy Efficient flow routing and scheduling}

\subsection{Flow Scheduling}

From the perspective of link energy efficiency, exclusive routing is more efficient than fair sharing routing and flow reservation. For example, we show the bandwidth allocation for 3 flows on a link in Fig. 1, where different colored sections denote
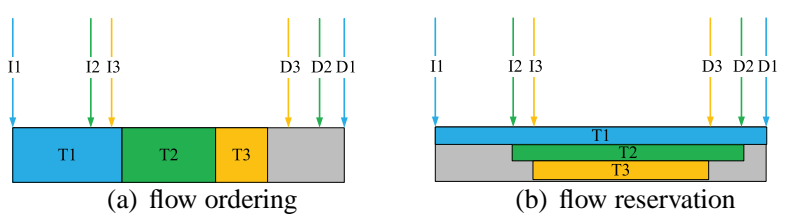

(b) flow reservation

Figure 1: An example of deadline flows. There are 3 flows of different release time $I_{1}<I_{2}<I_{3}$ and deadline $D_{1}>D_{2}>D_{3}$ sharing the same link. (a) shows a feasible sequence of flows with exclusive routing is $T 1, T 2, T 3$ as the deadline requirement can be fulfilled, while (b) shows another feasible scheduling that each flow is guaranteed to have a reserved bandwidth to finish before deadline. the flows' bandwidth allocation and the grey section denotes unused bandwidth. The idle link can sleep for energy saving. For network resource allocation methods, such as fair sharing flow scheduling and flow reservation, the bottleneck link will limit the flows' bandwidth while other links are not fully utilized. For flow reservation, each flow possesses a reservation of the minimal bandwidth on the path. As shown in Fig. 1(b), when the gaps are not long enough to serve any unreserved flow, the corresponding links will not be fully utilized. With exclusive routing, Fig. 1(a) shows a feasible allocation, a sequence of flows with an order of flow priority, which depends on flow deadline or flow size. By contrast, the flow scheduling in [28] is designed with abused preemptions, which provides unstable scheduling results.

\subsection{Energy of flow scheduling}

For a DCN network, we present all switches by $V$ and the adjacent switches by an adjacent matrix $A$, where $a_{i, k}=1$ if $v_{i}$ is connected to $v_{k}$, and $a_{i, k}=0$ if not. We denote a DCN flow with a 3-tuple flow model by $f_{j}=\left(t_{j}^{\text {arrival }}, t_{j}^{\text {deadline }}, A_{j}\right)$ for the arrival time, deadline and flow size. For each deadline constrained flow $f_{j}$, it will start transmission at $t_{j}^{\text {start }}$ and finish at $t_{j}^{\text {end }}$. For the switches $v \in P_{j}$ of the routing path $P_{j}$,

$$
\delta_{j, v}(t)=1, t_{j}^{\text {start }}<t<t_{j}^{\text {end }}
$$

which can be used to denote the path of a flow. Since that DCN traffic is always in a single path, the flows on any given link between two switches $v_{i}$ and $v_{k}$ are,

$$
F_{v_{i}, v_{k}}=\left\{f_{j} \mid \delta_{j, v_{i}}(t)=1 \& \& \delta_{j, v_{k}}(t)=1, \forall v_{i}, v_{k} \in V\right\}
$$

As flows will not share links with each other in exclusive routing, the active switch duration will be,

$$
T_{i}=\sum_{j}\left(t_{j}^{e n d}-t_{j}^{s t a r t}\right) \delta_{j, v_{i}}(t)
$$

And for any link between the adjacent switches $v_{i}$ and $v_{k}$, $a\left(v_{i}, v_{k}\right)=1$ is used to denote the adjacent relation, we can get the active duration of this link, denoted by,

$$
T_{v_{i}, v_{k}}=\sum_{j}\left(t_{j}^{e n d}-t_{j}^{s t a r t}\right) \cdot \delta_{j, v_{i}}(t) \cdot \delta_{j, v_{k}}(t) \cdot a_{i, k}
$$

For any feasible solution, the overall energy is,

$$
E=\sum_{v_{i} \in V}\left(F_{i} \times T_{i}+\sum_{v_{k} \in P(i)} D_{i} \times T_{v_{i}, v_{k}}\right)
$$

As we consider exclusive routing, any feasible solution will make full use of active ports. However, not all ports of active switches will be utilized. The utilization of an active switch is in a highly significant correlation with the number of active ports. Therefore, energy efficient solutions should increase the switch utilization. 


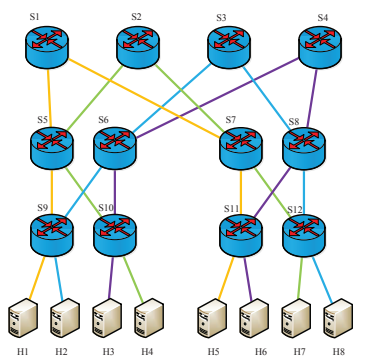

(a) A harmonic flow set

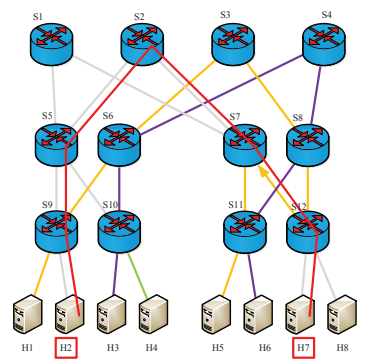

(b) Flow preemption
Figure 2: Example of flow preemption in a partial 4-ary Fat-Tree. (a) shows an harmonic flow set that achieves optimal energy efficiency, while a red flow preempt links of the flow set in (b).

\subsection{Problem Formula and Solutions}

As the power of the fixed and the dynamical part to be $\mathrm{F}$ and $\mathrm{D}$ for all network elements, the energy efficiency problem with the Objective Function (OF) Eq. 7 can be formulated as a linear programming in the following form,

$$
\text { minimize : } F \cdot \sum_{i \in V} T_{i}+D \cdot \sum_{v_{l}, v_{m} \in V} T_{v_{l}, v_{m}}
$$

subject to :Eq. 4, Eq. 5,Eq. 6, and

$$
\delta_{j, v}(t) \in\{0,1\}
$$

The best solution to the above problem consists of the path and the start time for each flow, which makes full use of the active switches with links in maximum utilization. The selected paths of the existing flows will contribute to the minimum energy consumption, which can be calculated with Eq. 7 by summing the working durations of active switches and ports.

A simple greedy method can select the best solution by calculating the network energy of all feasible solutions [26]. However, this method should consider $k_{1} \times k_{2} \ldots \times k_{n}$ solutions for flows $f_{1}, f_{2}, \ldots, f_{n}$ with the corresponding $k_{1}, k_{2}, \ldots, k_{n}$ paths for each flow. As the feasible paths for each flow is constrained to a small number, we can assume that the number of feasible paths is a constant, i.e. $k_{i}=k$, and then the greedy method will be executed in $O\left(k^{n}\right)$ time, which has exponential computational complexity respect to the number of flows.

So for the scalable traffic volume, we have to design a heuristic algorithm to solve the problem. The main idea is to aggregate deadline-constrained and port-monopolized flows into a minimal set of switches. Assuming all links have equal capacity, each link serves no more than one flow at a time. If there is no routing path available, flows with shared links will have to wait until other flows have finished.

\subsection{Energy Efficient solutions}

In this section, we illustrate our method of energy efficien$\mathrm{t}$ network resource allocations in DCN. Given a set of 4 flows $f_{1}: H 1 \rightarrow H 5, f_{2}: H 2 \rightarrow H 8, f_{3}: H 3 \rightarrow H 6, f_{4}: H 4 \rightarrow H 7$, exclusive routings in Fig. 2(a) allocate 12 switches and 40 ports to transfer 4 flows simultaneously, which brings a maximum of network energy efficiency, with the network energy $12 F+40 D$

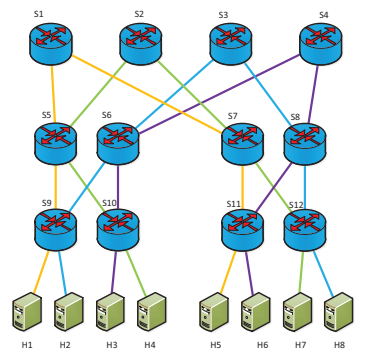

(a) non-preemptive routing, $\mathrm{t}$

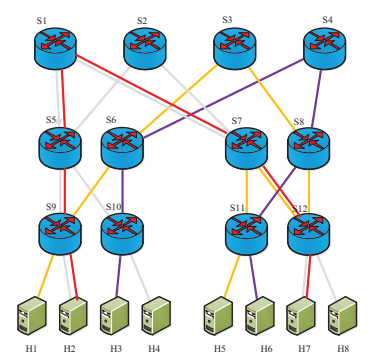

(c) preemptive routing, $\mathrm{t}$

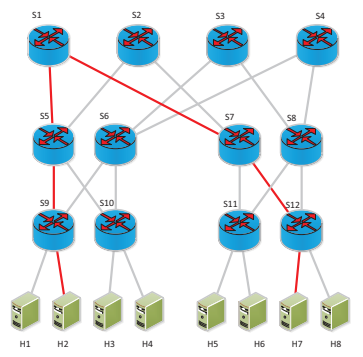

(b) non-preemptive routing, $\mathrm{t}+1$

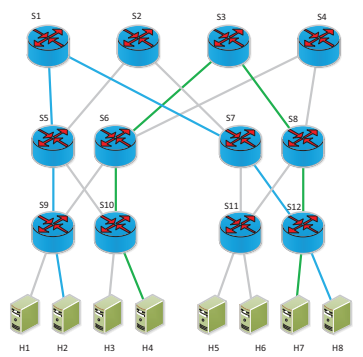

(d) preemptive routing, $\mathrm{t}+1$
Figure 3: Example of flow routing and schedule in a partial 4-ary Fat-Tree. The 5 flows are routed in two phases, at time $t$ and $t+1$.(a) and (b) show two phases of non-preemptive routing, an harmonic flow set at time t achieves optimal energy efficiency, (c) and (d) show preemptive routing, where the red flow preempt links in the first phase.

and the network throughput of 4 flows per unit time. We define these 4 flows a harmonic set of flows, as they can be transferred with network devices in full utilization. And now add a new flow $f_{5}: H 2 \rightarrow H 7$ to the above flow set, with priority higher than other 4 flows, the network with exclusive routings will transfer the $f_{5}$ preemptively along the red path, as shown in Fig. 2(b). As the flow $f_{5}$ preempts paths of $f_{2}$ and $f_{4}$, the flow $f_{1}$ can still be routed simultaneously in another path $H 2, S 9, S 6, S 3, S 8, S 12, S 7, S 11, H 5$, therefore, the network energy will be $11 F+36 D$ and network throughput is 3 per unit time for the flow $f_{1}, f_{3}$, and $f_{5}$.

Assuming that there are 5 unit flows arriving at the same time in the same network, a non-preemptive routing solution will transfer the 5 flows in two unit times. In the first round, 4 flows transmitted with paths distributed as shown in Fig.3(a), which consists of 12 switches and 40 ports, and in the second round, another flow is transmitted along the path shown in Fig.3(b), which consists of 5 switches and 10 ports. Summing the active time of switches and ports, we calculate the network energy of this solution is $17 F+50 D$. With preemptive routings, a feasible solution will transfer the 5 flows in two rounds. In the first round, there are 11 switches and 32 ports as shown in Fig.3(c), and in the second round, there are 9 switches and 20 ports as shown in Fig.3(d). Therefore, the network energy for transmitting these 5 flows preemptively is $20 F+52 D$. Comparing these two solutions, the preemption routing will ruin the harmonic set of the flows and break the optimal energy combination of flows leading to more energy consumption.

As flows are deadline constrained, we can combine flows with similar deadlines into harmonic set and assign it a higher 
priority in the flow scheduling. For example, we can schedule $f_{5}$ individually apart from other flows by forwarding it after the other 4 flows, as the 4 flows are the deadline of $f_{5}$ is longer than 2 unit time. This scheduling is based on the fact that flows will be consolidated into time slices of minimal active links.

In EXR, flow scheduling will preemptively schedule flows according to the flow priority, such as the flow deadline and flow size. For energy efficiency, EXR greedily and preemptively chooses a path with the minimal amount of sleeping switches, which ignores the energy of ports.

In our energy efficient method, we will consolidate flows with similar flow deadlines into harmonic flow sets. The challenge in designing our algorithm is that all queued flows will compete for links with different available time, which will make it hard to find a harmonic set of flows for the duration. As an extreme case, the scheduling will became a greedy scheduling if links are available one by one.

\section{Bandwidth-aware Energy Efficient Routing algorithm with SDN}

In this section, we design a flow scheduling and routing algorithm based on SDN controller, which manages SDN switches using OpenFlow protocol and communicates with servers via the Northbound APIs in a centralized way. Since all flows are scheduled by the same SDN Controller, it does not need clock synchronization for servers to correct flow launching. When no flow exists in a link, the corresponding two ports of this link can be put into sleep mode. When all the ports of an OpenFlow switch are asleep, this switch can be put into sleep. As a result, it is able to achieve high energy benefit.

The proposed algorithm, Bandwidth-aware Energy Efficient Routing algorithm with SDN (BEERS) can cooperate with servers and SDN switches to achieve the OF in Eq. (8). The BEERS schedules traffic flows into active and queued status. The active flows can immediately start along as the SDN controller can find and assign a feasible path. The queued flows will wait until the assigned paths are not in-use.

The BEERS is designed as a SDN controller application with northbound and southbound interfaces, the former will receive the information about flow size and flow deadline, while the later can monitor network status and install flow entries. Before a host starts a new flow, it will send a flow transfer request to the SDN controller, and BEERS on the controller will find a route and start the flow immediately or schedule the flow later. The BEERS can calculate the flow transfer duration with acknowledgement of flow requests and link utilizations.

\subsection{BEERS}

The main procedure of our scheduling algorithm is shown in Alg. 1 with the input of network topology G, existing flows F, and new flows. When a new flow $f_{i}$ arrives, BEERS will parse the flow with flow size $w_{i}$, release time $I_{i}$ and deadline $D_{i}$ from the flow request, and then decide the routing path and the start time of each flow. As the SDN controller monitors the flow size and flow rate of network flows in real time, BEERS can make full use of the acknowledgement of the network traffic distribution.

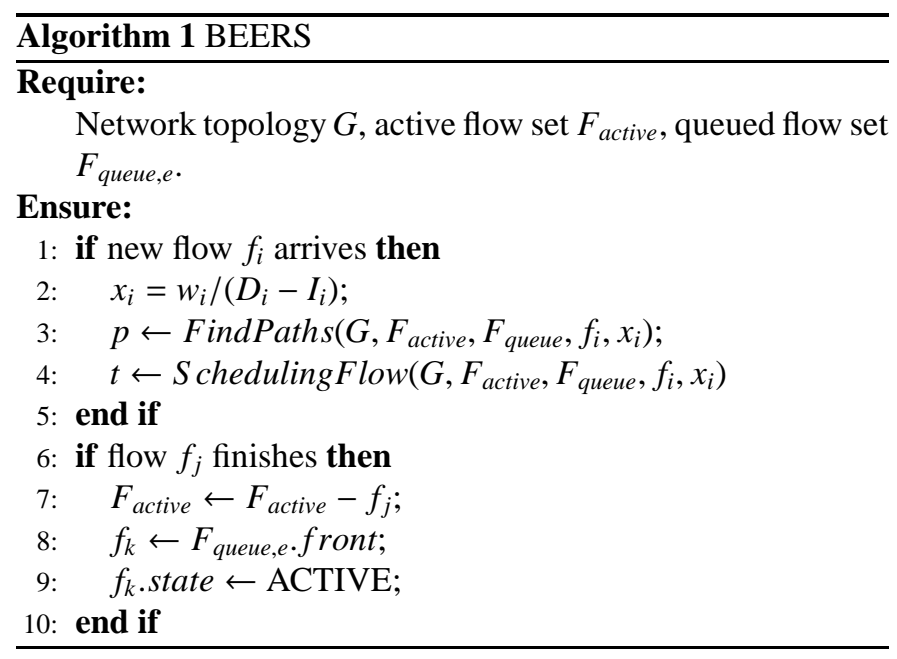

When the SDN controller receives a request of a new flow, BEERS will first transform the deadline and flow size into guaranteed flow rate $x_{i}$. And then the FindPath method searches for the flow paths with minimum links. Each flow has multiple paths in rich-connected DCN topologies. After that, the SchedulingFlow method will determine the start time of each flow that contributes to a minimal energy consumption. And when existing flow ends, the BEERS will remove the flow from the active flow set and start the next flow in the queue.

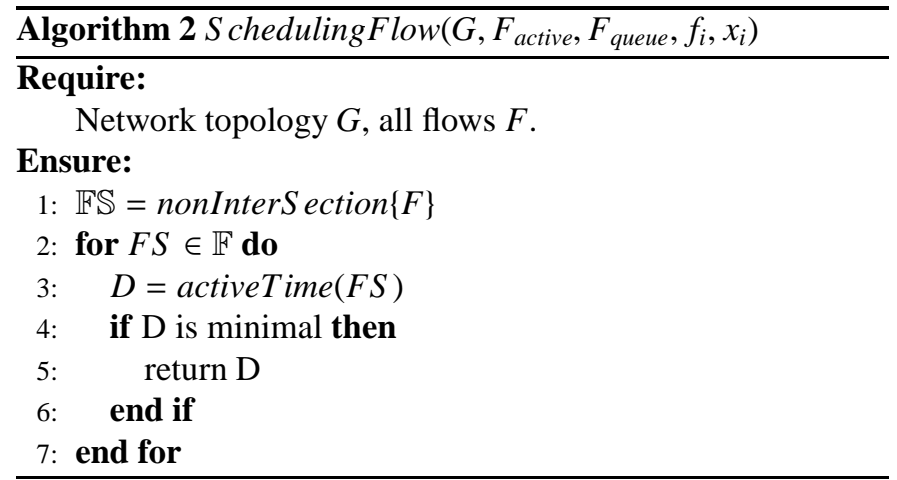

The Scheduling Flow method will schedule all flows' starting time for a less energy consumption. The flows in $F$ can be divided into several sets of flows $F S$ that each flow set contains no shared links. We denote all possible divisions of flows by $\mathbb{F} \mathbb{S}, F S \in \mathbb{F} S$, which can be searched by the nonInterS ection method. For each flow division $F S$, the activeTime method is used to count the running time of the active switches and active links for the flow sets according to Eq.(7). For all possible flow divisions, we will select the one with the minimum active time, which is the solution for Eq.(8).

In the solution with the minimum energy consumption, the flows are transferred along the selected path at selected period. As the typical DCN topologies provide rich connections, each flow will have multiple paths available, so when BEERS chooses routing paths for new flows, it may get 2 cases. 
1. When the new flow has a path that contributes to the minimum energy consumption, BEERS will start the flow immediately.

2. When the new flow has a path that has been used by existing flows, the flow should start later as it will be added into the queued flow set.

When the flow request is larger or much smaller than the network capacity, BEERS will not be able to schedule flows optimally. When a new flow joins the network, the SDN controller can calculate the fair share flow rate according to the fair sharing scheme. The flows with higher flow rate will preferentially have available paths.

1. When the new flow request is much lower than the network capacity, the flow will be consolidated into links, similar to the shortest path routing.

2. When the new flow request is much higher than the network capacity, the flow will be distributed into separate paths as possible.

Defining the power utilization as the ratio of the actual throughput over the maximum throughput of active time, the optimal flow scheduling will maximize the utilization of the active switches and active links.

\subsection{Algorithm Analysis}

On basis of the EXR flow scheduling method, the SDN controller can be designed to schedule the flow start time according to the acknowledgement of the release time, deadline, link bandwidth, etc. We find that there are several problems in the original EXR flow scheduling method.

Firstly, the SDN controller first chooses an available path, and then chooses the preemptive path to fulfill the deadline requirement for each new flow. If there are more than one alternative path, the one with the fewest sleeping switches will be chosen, which may be longer than the shortest path in FSR.

Secondly, the preemption is carried out according to the priority rather than deadline, leaving less potential of combinational optimization. When the flow preemption is determined by deadline strictly, it may only achieve energy efficiency at local level rather than global level. Although the flow scheduling with preemption can make high priority flows to monopolize the shared links and makes the unshared links into sleep, the number of flows on the preemptive path is not considered, which will cause useless transition time.

In BEERS, we schedule new flows into queues based on the deadline constraint, and carry out the combinational optimization in the queued flows. The network constrained flows can be scheduled by the flow aggregation methods, when we transform the flow size and flow deadline into an entry of the traffic matrix; however for some flows, such as the real-time streaming, the flow rate cannot be transformed into tuples of flow size and deadline. Compare with flow aggregation methods, BEERS can optimize the overall energy in time dimension, as the flows can be scheduled once the queued flows are not optimally preallocated in the incremental approach.
In our method, we extend the EXR flow scheduling by considering the overall time of active switches. From the perspective of time dimension, flows will share the same link with other flows with different monopolizing time. Similar to the flow consolidation with flow correlation, our method can group the flows without shared links, and then consolidate the flow groups into a minimal topology.

\section{Performance Evaluation}

In this section, we present the simulation results to evaluate BEERS. We simulated BEERS and the baseline routing algorithms under typical DCN traffics in OMNeT++4.2. The routing algorithms are implemented as the experiment control modules on an OpenFlow integrated platform [42].

In the existing platforms of energy simulation, the work [43] is a TCP/IP support packet based simulator with NS2 that counts the total power consumption of servers, switches and links. Considering server workloads, the simulator [44] provides computational intensive, the data intensive and the balanced DC load. In our platform, the DCN traffic generator is implemented as a TCP application with SDN northbound supported, inherited from the TCP/IP supported INET framework. The SDN controller is implemented with a northbound interface for arriving flow requirements. The network energy is measured by counting the running time of switch linecards and switch ports. The power of both linecards and ports is fixed [21], for instance, the fixed power is $36 \mathrm{~W}$ for WS-C2960G-8TC-L and $72 \mathrm{~W}$ for WS-C2960G-24TC-L, while the port power is $1 \mathrm{~W}$. And for BCube, the NIC of servers act as network devices, with the power $10 \mathrm{~W}$ for a typical 4-port NIC as an example.

\subsection{Simulation Setup}

To build typical DCN topologies, we set up a 4-ary Fat-Tree built by 4-port switches and a BCube network built by 4-port switches and servers with 4-port NIC. The flows were generated randomly by each host choosing a random host and a random flow size following an exponential distribution with mean 64MB. For energy model of network devices, we set $36 \mathrm{~W}$ for fixed power of active switches, $10 \mathrm{~W}$ for 4 -port NIC, and $1 \mathrm{~W}$ for active ports. And the network switches and ports were sleep in 2 seconds and 10 milliseconds separately.

To generate typical DCN traffic, we set the TCP clients distributed on hosts that start flows to a randomly selected destination with flow intervals of a given inter-arrival time. There are three traffic patterns,. In the first pattern, flows are prone to be generated between nearby nodes for the DCN applications, such as the nodes under the same switch or pod. In the second pattern, flows start between any randomly selected nodes. And in the third pattern, there are aggregation flows from multiple senders to a receiver, such as the map-reduce applications. We set the flow size of large flows arranges from $128 \mathrm{M}$ to $1 \mathrm{G}$ byte, and took the flow arrival time as the network workload. Considering the network workload capacity, we set the average inter-arrival time for flows a little longer than the transmission time of flows, for example 1.5 times or 1.125 times. 


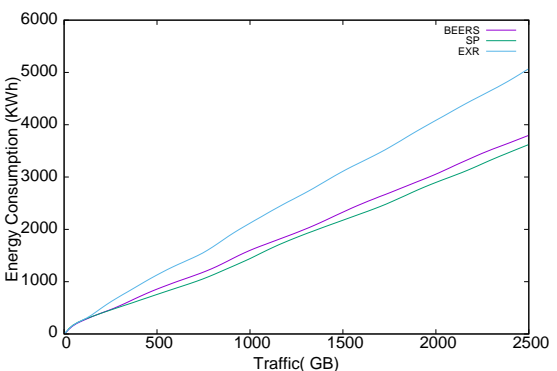

(a) Energy

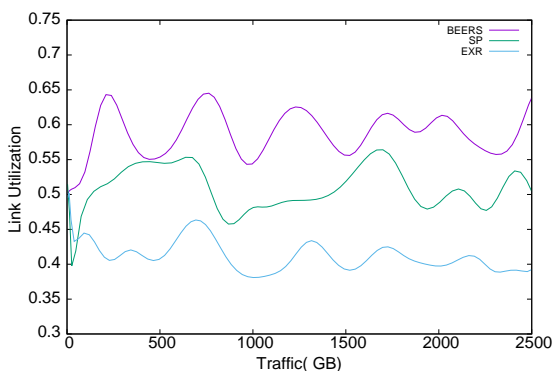

(b) Link Utilization

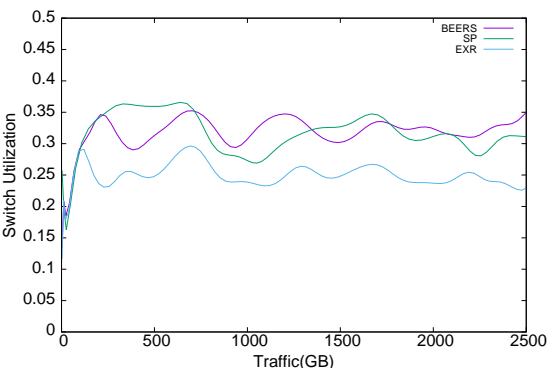

(c) Switch Utilization

Figure 4: Performance for aggregation traffic in FatTree network.

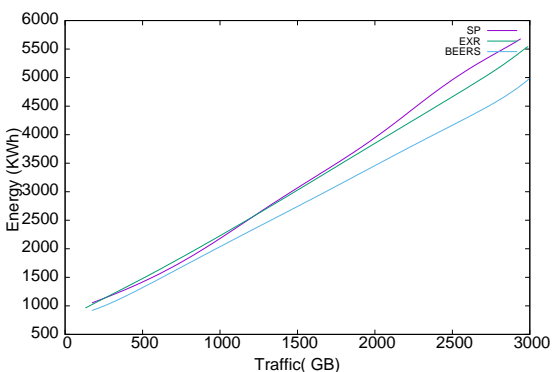

(a) Energy

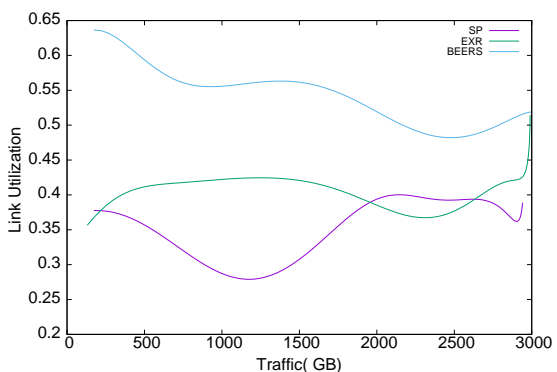

(b) Link Utilization

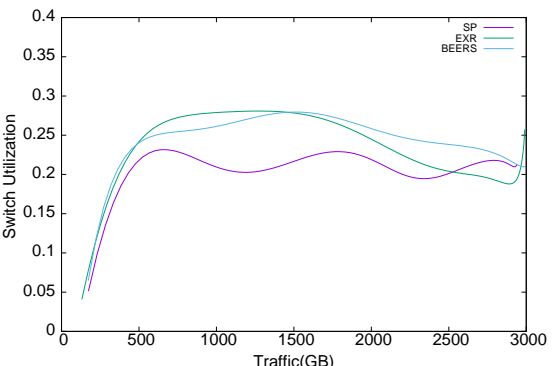

(c) Switch Utilization

Figure 5: Performance for aggregation traffic in BCube network.

As the baseline methods, we also implemented the shortest path (SP) routing and EXR routing as two routing modules. In SP routing, all flows share the links with same priority. In EXR routing, flows are routed to available path first and then scheduled according to priority.

The exclusive flow scheduling will not share links with other flows at the same time. For static routings, each flow has a static routing path, exclusive flow scheduling will queue flows with shared links with arrival order. When the flow scheduler allows preemptive routing, the flow with higher priority can monopolize a path that may be transferring other flows of lower priority. For fixed routing method, the traffic across switches are fixed, and as a result, different flow arrivals consume the same link energy for the same set of flows. When the flow path is determined dynamically, flows will be routed dynamically through path$\mathrm{s}$ with available resources, and the amount of active switches will depend on the shared switches between flows. As a result, different flow scheduling methods will lead to different energy consumption of switches. In an ideal case of energy efficient flow scheduling, flows will utilize switches and links with the minimum active time.

To evaluate the performance of different routing algorithms, we define new metrics of the switch utilization ratio and the link utilization ratio, the former is the real-time traffic of active switches over the maximum traffic capacity of active switches, and the later denotes the real-time traffic of active links over the maximum traffic capacity of active links. Although the flow completion time is important for typical DCN traffic, we set deadline for each flow in our evaluation, so that all completed flows can end with the acceptable transmission time.

\subsection{Performance for Aggregation Flows in FatTree}

In a 4-ary FatTree DCN, we generate groups of traffic data with the overall traffic volume varying from $0 G B$ to $2500 G B$, with each group including flows from several nodes to another node. The average inter-arrival time is set to be 1.125 times of the average flow transmission time. Then we get the results of energy efficiency and the network utilization. In Fig. 4(b), the link utilization ratios are above $40 \%$, which means the traffic is a heavy workload for this network. The link utilization and switch utilization of BEERS are higher than EXR (Fig. 4(b) and Fig. 4(c)), however, the energy consumption is higher than EXR (in Fig. 4(a)). When we look into the results of BEERS and EXR, we find that about $20 \%$ flows in the BEERS are transferred with flow rate $65 \%$ of the link capacity while all flows in the EXR are transferred with flow rate $99 \%$. This happens when some flows are not transferred with the full speed that the network can support. This result infers that high utilization does not mean high efficiency.

To evaluate the performance of flow transmission time, we run simulations with the flow size varying from $30 \mathrm{MB}$ to $180 \mathrm{M}-$ B. We denote the transmission time when the flow is transmitted solely by $T S$, and set the flow deadline $50 \%$ longer than $T S$. The results in Fig. 9 shows the average flow transmission time normalized to $T S$. The flow transmission time is $10 \%$ to $30 \%$ longer in BEERS than that in EXR. This is because the EXR algorithm schedules flows in the deadline order, and achieves the shortest average transmission time when the flow size is less 


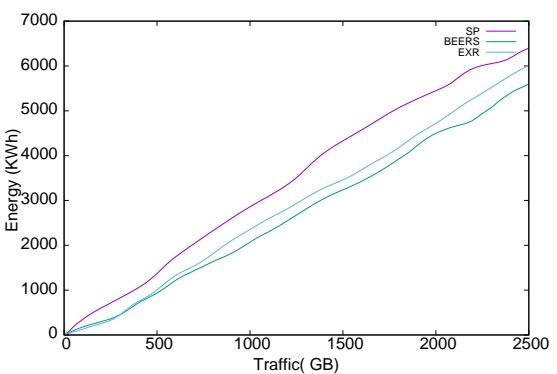

(a) Energy

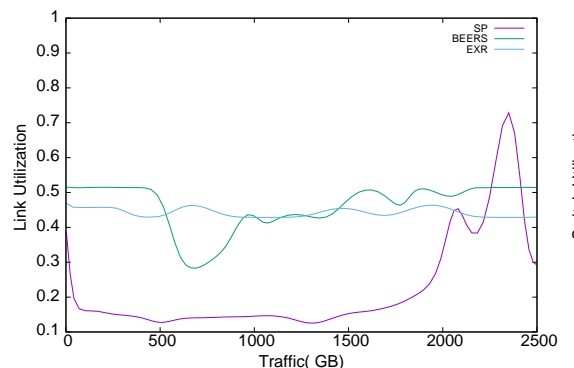

(b) Link Utilization

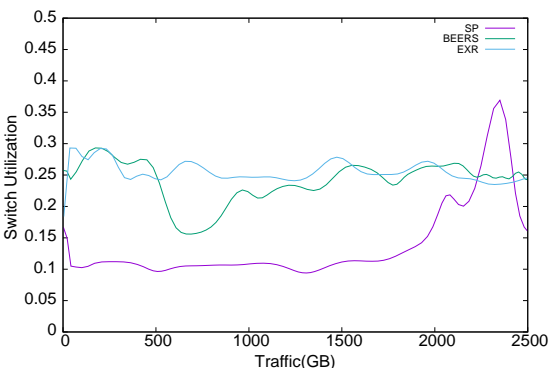

(c) Switch Utilization

Figure 6: Performance for random traffic with light network workload in FatTree network.

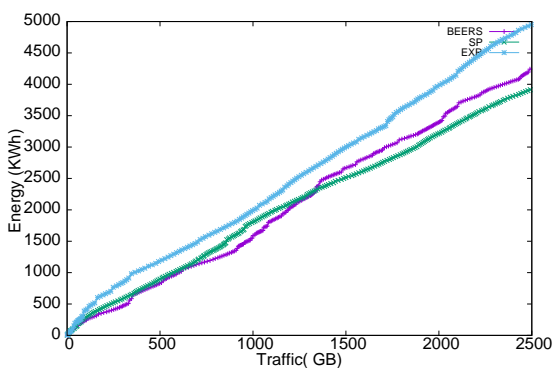

(a) Energy

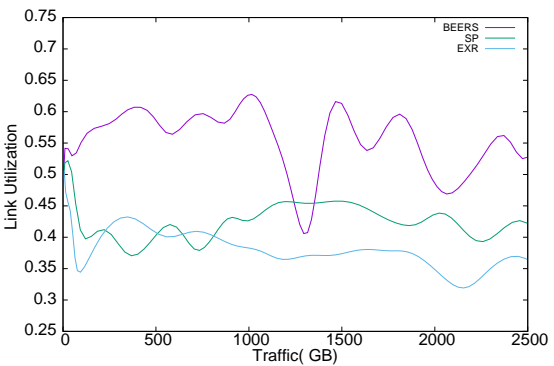

(b) Link Utilization

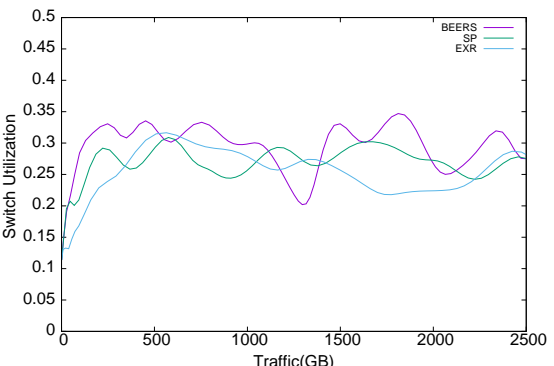

(c) Switch Utilization

Figure 7: Performance for random flows with heavy network workload in FatTree network.

than 120MB. The BEERS algorithm schedules flows for energy efficiency under the constrains of the flows' deadline, which brings flow transmission time no more than $1.4 \mathrm{TS}$. This result is acceptable as it is meet the deadline constraints $50 \%$. Therefore, the BEERS increases the average transmission time without negatively impacting the performance of the flows from the perspective of flow deadline. In the following simulations, we set flow deadline to be $50 \%$ longer than $T S$, and focus on the evaluation of the energy consumption.

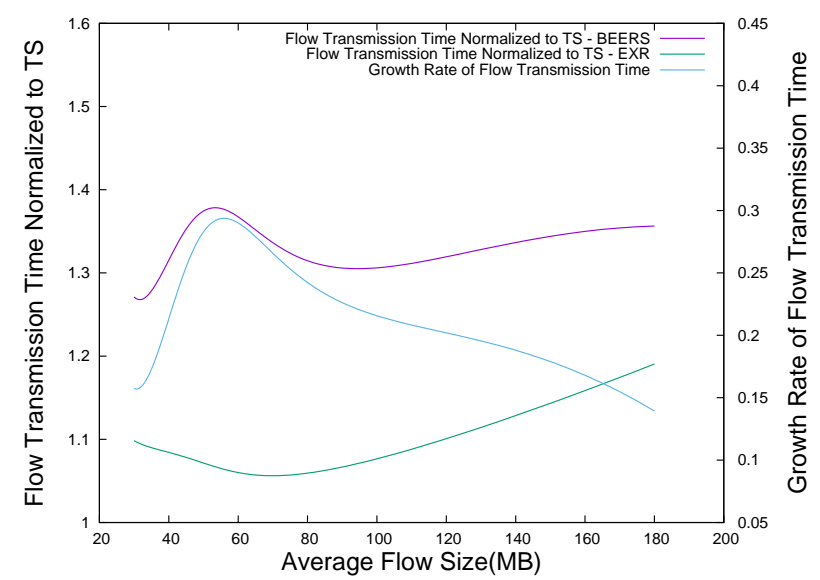

Figure 9: The flow Transmission Time evaluation under different traffic generating rate in FatTree network. The left axis is the average flow transmission time normalized to $T S$, while the right axis is the Growth Rate of flow transmission time for the BEERS algorithm.

\subsection{Performance for Aggregation Flows in BCube}

In a BCube DCN, we generate a random traffic to simulate the typical aggregation applications, such as MapReduce data shuffling. The overall traffic data ranges from 0 to 3000GB. The result in Fig. 5 shows that the energy consumption with BEERS is lower than the one with EXR, and the energy consumption with EXR is lower than the one with SP for most of the traffic. This is because the BEERS and EXR can make better use of links, and the link utilization with BEERS are higher than that with EXR, as shown in Fig. 5(b). The Fig. 5(c) shows that there is no obvious difference between the utilization ratio with BEERS and the one with EXR. Combined with the results of link utilization, we can infer that the EXR exploits more alternative paths, with the sacrifice of a lower probability of the reverse link, so the link utilization ratio with EXR is lower than $50 \%$, much lower than the one with BEERS.

\subsection{Performance for Random Flows in FatTree}

In Fig. 6, we set a light network workload where the average inter-arrival time of randomly generated flows is 1.5 times of the average flow transfer time. The results in Fig. 6(a) shows that the energy consumption of BEERS and EXR are lower than SP. As shown in Fig. 6(b), the links are not well utilized in SP. As the SP cannot make use of multipath routings, the flows sharing links with other flows are constrained by the flow completion on the shared links rather than the link capacity of the unshared links. The shared links are easily to be fully utilized while the other links will be in low utilization, so the overall link utilization in SP is lower than the other two methods. The 


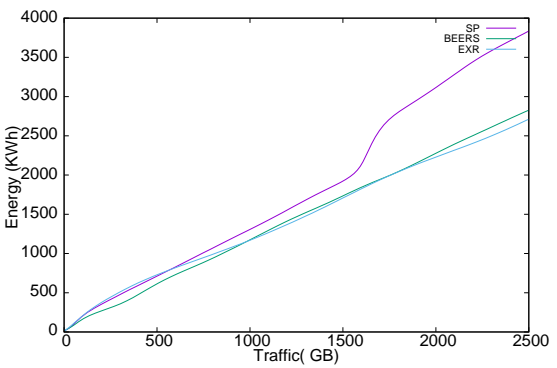

(a) Energy

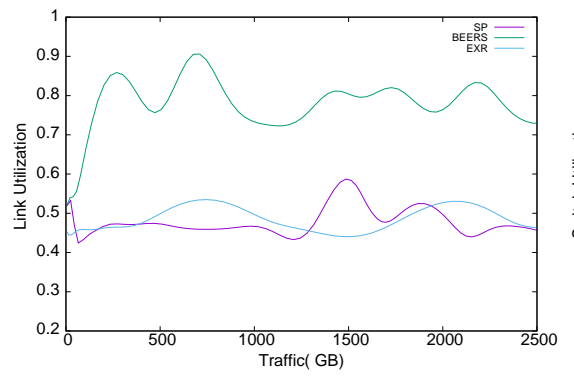

(b) Link Utilization

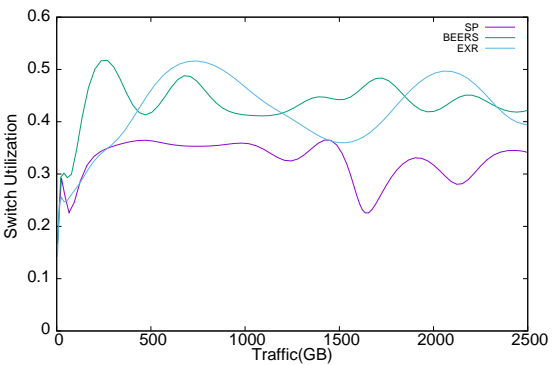

(c) Switch Utilization

Figure 8: Performance for uniform traffic in FatTree network.

utilization of any link consist of bi-direction bandwidth, so even when a flow monopolize a link, the link utilization may be a little bit higher than $50 \%$, as the reverse direction only consists of control messages, which may be nearly idle.

For the heavy network workload, we set the average interarrival time of flows to be 1.125 , which influences the link utilization and the switch utilization directly, The switch utilization with SP shown in Fig. 7(c) is more than two times of the one with SP in Fig. 6(c). The Fig. 7(a) shows that there are fewer potentials to save energy when the network is nearly saturated. When the traffic is from 0 to $600 \mathrm{~GB}$, the energy consumption with BEERS is similar to the one with SP. As traffic increase between 600GB to 1300GB, BEERS will consume less energy than SP, And after 1300GB, BEERS will consume more energy than SP. In the result, the energy consumption with EXR is more than the other two in any cases. Therefore, the nonpreemptive exclusive routing will consume more energy than SP and BEERS.

In Fig.7(b), we measure the average link utilization with Bezier curve. In this plot we measure the link utilization by dividing the traffic on both direction of the link. As the exclusive routing guarantees that each active flow can exclusively use the links along the path, the flows will increase the rate step by step, such as TCP, and the utilization of links from the source to the destination will be nearly in full utilization. However, this will approach to $50 \%$ when we consider the bi-direction of each link, as the reverse links will still be in the low utilization. From the results of random traffic, the average utilization of links in BEERS is more than $50 \%$ for most of the traffic. In Fig. 7(c), the BEERS has the most switches utilization for most cases.

As a worst case scenario, we generate the random traffic with the most flows across the core switches, and get the results in Fig. 8. In Fig. 8(a), the BEERS method has slightly lower energy consumption than EXR even when it achieves higher link utilization and higher switch utilization for the traffic from 0 to 1000GB. As the traffic becomes higher than 1000GB, the energy consumption with BEERS is similar with the one with EXR. So the BEERS improves the link and switch utilization, however there is no obvious improvement for the energy consumption.

For the link utilization ratio of the our simulations, we take each link by a bidirectional link and consider the sum of individual bandwidth in bi-direction links, so the link utilization will approach $50 \%$ when a direction is totally monopolized by a flow. In Fig. 8(b), the link utilization ratio with BEERS can be about $80 \%$, while the one with the EXR or SP is about $45 \%$. This means that the BEERS can make better use of reverse links as well. In Fig. 8(c), the switch utilization ratio with BEERS or EXR is higher than the one with SP, because BEERS and EXR consider alternative paths. When the switch utilization is low at the traffic $1600 \mathrm{~GB}$, the switch utilization of SP is about $20 \%$, the link utilization of SP is about $50 \%$, while the difference between the energy consumptions with BEERS and EXR increases dramatically. This may happen when more paths are sharing the same links with SP, as the link utilization and switch utilization both decrease after 1600GB.

\section{Conclusion and Future Work}

In this paper, we propose BEERS, a new energy efficien$t$ routing algorithm with SDN for data center networks. The BEERS algorithm can schedule flows to the queues of each link to minimize the energy for the given volume of data center traffic. The simulation results verify that BEERS can make full use of active switches for less energy cost. As an extension work, the energy efficient flow scheduling for the DCN topologies built with variant links will be considered in the future, as we only consider typical DCN networks with identical links in BEERS. Also, we will consider a relaxed flow scheduling that can alleviate or avoid the TCP slow-start, as the deadline based flow scheduling does not insure that the network constrained flows with TCP slow-start can make use of exclusive paths. Besides, we will implement BEERS on real-world platforms built with Dell servers and SDN controllers, such as OpenDayLight [45] or ONOS [46].

\section{Acknowledgment}

This work was supported by the National Key Technology Research and Development Program of the Ministry of Science and Technology of China under Grant no. 2012BAH93F01, the Innovation Research Fund of Huazhong University of Science and Technology, No. 2015MS037 and the National Science Foundation of China under Grant no. 60803005.

[1] C. J. DOrazio, K. K. R. Choo, L. T. Yang, Data exfiltration from internet of things devices: ios devices as case studies, IEEE Internet of Things Journal PP (99) (2016) 1-1. doi:10.1109/JIOT.2016.2569094. 
[2] R. Jiang, R. Lu, K.-K. R. Choo, Achieving high performance and privacy-preserving query over encrypted multidimensional big metering data, Future Generation Computer Systems (2016) doi:10.1016/j.future.2016.05.005.

URL http://www.sciencedirect.com/science/article/pii/ S0167739X16301157

[3] X. Liu, R. Choo, R. Deng, R. Lu, J. Weng, Efficient and privacypreserving outsourced calculation of rational numbers, IEEE Transactions on Dependable and Secure Computing PP (99) (2016) 1-1. doi:10.1109/TDSC.2016.2536601.

[4] V. Odelu, A. K. Das, Y. S. Rao, S. Kumari, M. K. Khan, K.-K. R. Choo, Pairing-based cp-abe with constant-size ciphertexts and secret keys for cloud environment, Computer Standards \& Interfaces (2016) -doi:http://dx.doi.org/10.1016/j.csi.2016.05.002.

URL http://www.sciencedirect.com/science/article/pii/ S0920548916300368

[5] O. Osanaiye, K.-K. R. Choo, M. Dlodlo, Distributed denial of service (ddos) resilience in cloud: Review and conceptual cloud $\{\mathrm{DDoS}\}$ mitigation framework, Journal of Network and Computer Applications 67 (2016) 147 - 165. doi:http://dx.doi.org/10.1016/j.jnca.2016.01.001. URL http://www.sciencedirect.com/science/article/pii/ S1084804516000023

[6] Y. Yang, H. Zhu, H. Lu, J. Weng, Y. Zhang, K.-K. R. Choo, Cloud based data sharing with fine-grained proxy re-encryption, Pervasive and Mobile Computing 28 (2016) 122 - 134, special Issue on Big Data for Healthcare; Guest Editors: Sriram Chellappan, Nirmalya Roy, Sajal K. Das and Special Issue on Security and Privacy in Mobile Clouds Guest; Editors: Sherman S.M. Chow, Urs Hengartner, Joseph K. Liu, Kui Ren. doi:http://dx.doi.org/10.1016/j.pmcj.2015.06.017.

URL http://www.sciencedirect.com/science/article/pii/ S1574119215001248

[7] L. Li, R. Lu, K. K. R. Choo, A. Datta, J. Shao, Privacy-preservingoutsourced association rule mining on vertically partitioned databases, IEEE Transactions on Information Forensics and Security 11 (8) (2016) 1847-1861. doi:10.1109/TIFS.2016.2561241.

[8] N. H. Ab Rahman, N. D. W. Cahyani, K.-K. R. Choo, Cloud incident handling and forensic-by-design: cloud storage as a case study, Concurrency and Computation: Practice and Experience (2016) n/an/adoi:10.1002/cpe.3868.

URL http://dx.doi.org/10.1002/cpe. 3868

[9] N. H. A. Rahman, W. B. Glisson, Y. Yang, K. K. R. Choo, Forensic-bydesign framework for cyber-physical cloud systems, IEEE Cloud Computing 3 (1) (2016) 50-59. doi:10.1109/MCC.2016.5.

10] Q. Do, B. Martini, K. K. R. Choo, A cloud-focused mobile forensics methodology, IEEE Cloud Computing 2 (4) (2015) 60-65. doi:10.1109/MCC.2015.71.

[11] D. Quick, K.-K. R. Choo, Google drive: Forensic analysis of data remnants, Journal of Network and Computer Applications 40 (2014) 179 - 193. doi:http://dx.doi.org/10.1016/j.jnca.2013.09.016.

URL http://www.sciencedirect.com/science/article/pii/ S1084804513002051

[12] B. Martini, K.-K. R. Choo, Cloud storage forensics: owncloud as a case study, Digital Investigation 10 (4) (2013) 287 - 299. doi:http://dx.doi.org/10.1016/j.diin.2013.08.005.

URL http://www.sciencedirect.com/science/article/pii/ S1742287613000911

[13] D. Quick, K.-K. R. Choo, Forensic collection of cloud storage data: Does the act of collection result in changes to the data or its metadata?, Digital Investigation 10 (3) (2013) 266 - 277. doi:http://dx.doi.org/10.1016/j.diin.2013.07.001.

URL http://www.sciencedirect.com/science/article/pii/ S1742287613000741

[14] B. Martini, K. K. R. Choo, Remote programmatic vcloud forensics: A six-step collection process and a proof of concept, in: 2014 IEEE 13th International Conference on Trust, Security and Privacy in Computing and Communications, 2014, pp. 935-942. doi:10.1109/TrustCom.2014.124.

[15] N. D. W. Cahyani, B. Martini, K.-K. R. Choo, A. M. N. Al-Azhar, Forensic data acquisition from cloud-of-things devices: windows smartphones as a case study, Concurrency and Computation: Practice and Experience (2016) n/a-n/adoi:10.1002/cpe.3855

[16] K. Bilal, S. U. R. Malik, O. Khalid, A. Hameed, E. Alvarez, V. Wi- jaysekara, R. Irfan, S. Shrestha, D. Dwivedy, M. Ali, U. S. Khan, A. Abbas, N. Jalil, S. U. Khan, A taxonomy and survey on green data center networks, Future Generation Computer Systems 36 (2014) 189208, special Section: Intelligent Big Data ProcessingSpecial Section: Behavior Data Security Issues in Network Information PropagationSpecial Section: Energy-efficiency in Large Distributed Computing ArchitecturesSpecial Section: eScience Infrastructure and Applications. doi:10.1016/j.future.2013.07.006.

URL http://www.sciencedirect.com/science/article/pii/ S0167739X13001519

[17] M. Kassner, Data centers focus on pue in their quest to use electricity efficiently, Tech. rep. (2014).

URL http://www.techrepublic.com/article/ data-centers-focus-on-pue-in-their-quest-to-use-electricity-ef

[18] P. X. Gao, A. R. Curtis, B. Wong, S. Keshav, It's not easy being green, in: Proceedings of the ACM SIGCOMM 2012 Conference on Applications, Technologies, Architectures, and Protocols for Computer Communication, SIGCOMM '12, ACM, New York, NY, USA, 2012, pp. 211-222. doi: $10.1145 / 2342356.2342398$.

[19] A. Greenberg, J. Hamilton, D. A. Maltz, P. Patel, The cost of a cloud: Research problems in data center networks, SIGCOMM Comput. Commun. Rev. 39 (1) (2008) 68-73. doi:10.1145/1496091.1496103.

[20] D. Abts, M. R. Marty, P. M. Wells, P. Klausler, H. Liu, Energy proportional datacenter networks, SIGARCH Comput. Archit. News 38 (3) (2010) 338-347. doi:10.1145/1816038.1816004.

[21] M. Xu, Y. Shang, D. Li, X. Wang, Greening data center networks with throughput-guaranteed power-aware routing, Computer Networks 57 (15) (2013) 2880-2899. doi:10.1016/j.comnet.2012.12.012.

URL http://www.sciencedirect.com/science/article/pii/ S1389128612004161

[22] P. Mahadevan, P. Sharma, S. Banerjee, P. Ranganathan, A Power Benchmarking Framework for Network Devices, Springer Berlin Heidelberg, Berlin, Heidelberg, 2009, pp. 795-808. doi:10.1007/978-3-642-013997_62.

URL http://dx.doi.org/10.1007/978-3-642-01399-7\_62

[23] Y. Shang, D. Li, M. Xu, Energy-aware routing in data center network, in: Proceedings of the First ACM SIGCOMM Workshop on Green Networking, Green Networking '10, ACM, New York, NY, USA, 2010, pp. 1-8. doi:10.1145/1851290.1851292.

[24] Y. Yang, M. Xu, Q. Li, Towards fast rerouting-based energy efficient routing, Comput. Netw. 70 (2014) 1-15. doi:10.1016/j.comnet.2014.04.014.

[25] B. Heller, S. Seetharaman, P. Mahadevan, Y. Yiakoumis, P. Sharma, S. Banerjee, N. McKeown, Elastictree: Saving energy in data center networks, in: Proceedings of the $7^{\text {th }}$ USENIX Conference on Networked Systems Design and Implementation, NSDI'10, USENIX Association, Berkeley, CA, USA, 2010, pp. 17-17.

URL http://dl . acm.org/citation. cfm?id=1855711. 1855728

[26] D. Li, Y. Yu, W. He, K. Zheng, B. He, Willow: Saving data center network energy for network-limited flows, IEEE Transactions on Parallel and Distributed Systems 26 (9) (2015) 2610-2620. doi:10.1109/TPDS.2014.2350990.

[27] L. Wang, F. Zhang, K. Zheng, A. V. Vasilakos, S. Ren, Z. Li$\mathrm{u}$, Energy-efficient flow scheduling and routing with hard deadlines in data center networks, in: Distributed Computing Systems (ICDCS), 2014 IEEE $34^{\text {th }}$ International Conference on, 2014, pp. 248-257. doi:10.1109/ICDCS.2014.33.

[28] D. Li, Y. Shang, C. Chen, Software defined green data center network with exclusive routing, in: IEEE INFOCOM 2014 - IEEE Conference on Computer Communications, 2014, pp. 1743-1751. doi:10.1109/INFOCOM.2014.6848112.

[29] B. A. A. Nunes, M. Mendonca, X. N. Nguyen, K. Obraczka, T. Turletti, A survey of software-defined networking: Past, present, and future of programmable networks, IEEE Communications Surveys Tutorials 16 (3) (2014) 1617-1634. doi:10.1109/SURV.2014.012214.00180.

[30] A. D. Ferguson, A. Guha, C. Liang, R. Fonseca, S. Krishnamurthi, Hierarchical policies for software defined networks, in: Proceedings of the First Workshop on Hot Topics in Software Defined Networks, HotSDN '12, ACM, New York, NY, USA, 2012, pp. 37-42. doi: $10.1145 / 2342441.2342450$.

URL http://doi .acm.org/10.1145/2342441.2342450

[31] X. Wang, Y. Yao, X. Wang, K. Lu, Q. Cao, Carpo: Correlation- 
aware power optimization in data center networks, in: INFOCOM, 2012 Proceedings IEEE, 2012, pp. 1125-1133. doi:10.1109/INFCOM.2012.6195471.

[32] Y. Shang, D. Li, M. Xu, Greening data center networks with flow preemption and energy-aware routing, in: Local Metropolitan Area Networks (LANMAN), $201319^{\text {th }}$ IEEE Workshop on, 2013, pp. 1-6. doi:10.1109/LANMAN.2013.6528281.

[33] Y. Ding, X. Qin, L. Liu, T. Wang, Energy efficient scheduling of virtual machines in cloud with deadline constraint, Future Generation Computer Systems 50 (2015) 62 - 74, quality of Service in Grid and Cloud 2015. doi:http://dx.doi.org/10.1016/j.future.2015.02.001.

URL http://www.sciencedirect.com/science/article/pii/ S0167739X15000369

[34] J. Wu, Energy-efficient scheduling of real-time tasks with shared resources, Future Generation Computer Systems 56 (2016) 179 - 191. doi:http://dx.doi.org/10.1016/j.future.2015.05.012.

URL http://www.sciencedirect.com/science/article/pii/ S0167739X15001879

[35] R. K. Pal, I. Shanaya, K. Paul, S. Prasad, Dynamic core allocation for energy efficient video decoding in homogeneous and heterogeneous multicore architectures, Future Generation Computer Systems 56 (2016) 247 - 261. doi:http://dx.doi.org/10.1016/j.future.2015.09.018.

URL http://www.sciencedirect.com/science/article/pii/ S0167739X15002988

[36] M. Carabaş, P. G. Popescu, Energy-efficient virtualized clusters, Future Generation Computer Systems (2015) doi:http://dx.doi.org/10.1016/j.future.2015.10.018.

URL http://www.sciencedirect.com/science/article/pii/ S0167739X15003313

[37] J. Jiang, Y. Feng, J. Zhao, K. Li, Dataabc: A fast $\{A B C\}$ based energy-efficient live $\{\mathrm{VM}\}$ consolidation policy with data-intensive energy evaluation model, Future Generation Computer Systems (2016) -doi:http://dx.doi.org/10.1016/j.future.2016.05.013.

URL http://www.sciencedirect.com/science/article/pii/ S0167739X16301236

[38] G. Xu, B. Dai, B. Huang, J. Yang, Bandwidth-aware energy efficien$t$ routing with sdn in data center networks, in: Proceedings of the 2015 IEEE $17^{\text {th }}$ International Conference on High Performance Computing and Communications, 2015 IEEE $7^{\text {th }}$ International Symposium on Cyberspace Safety and Security, and 2015 IEEE $12^{\text {th }}$ International Conf on Embedded Software and Systems, HPCC-CSS-ICESS '15, IEEE Computer Society, Washington, DC, USA, 2015, pp. 766-771. doi:10.1109/HPCC-CSS-ICESS.2015.12.

[39] G. Ananthanarayanan, R. H. Katz, Greening the switch, in: Proceedings of the 2008 Conference on Power Aware Computing and Systems, HotPower'08, USENIX Association, Berkeley, CA, USA, 2008, pp. 7-7.

URL http://dl . acm.org/citation. cfm?id=1855610.1855617

[40] Cisco, Cisco nexus 2200 series fabric extenders data sheet, Tech. rep.

URL http://www.cisco.com/c/en/us/products/collateral/ switches/nexus-2000-series-fabric-extenders/data_ sheet_c78-507093.html

[41] IEEE p802.3az energy efficient ethernet task force.

[42] D. Klein, M. Jarschel, An openflow extension for the omnet++ inet framework, in: Proceedings of the $6^{\text {th }}$ International ICST Conference on Simulation Tools and Techniques, SimuTools '13, ICST (Institute for Computer Sciences, Social-Informatics and Telecommunications Engineering), ICST, Brussels, Belgium, Belgium, 2013, pp. 322-329.

URL http: //dl . acm.org/citation. cfm?id=2512734.2512780

[43] D. Kliazovich, P. Bouvry, S. U. Khan, Greencloud: a packet-level simulator of energy-aware cloud computing data centers, The Journal of Supercomputing 62 (3) (2012) 1263-1283. doi:10.1007/s11227-010-0504-1.

[44] A. Hammadi, L. Mhamdi, Review: A survey on architectures and energy efficiency in data center networks, Comput. Commun. 40 (2014) 1-21. doi:10.1016/j.comcom.2013.11.005.

[45] [link].

URL http://www. opendaylight.org/resources

[46] P. Berde, M. Gerola, J. Hart, Y. Higuchi, M. Kobayashi, T. Koide, B. Lantz, B. O'Connor, P. Radoslavov, W. Snow, G. Parulkar, Onos: Towards an open, distributed sdn os, in: Proceedings of the Third Workshop on Hot Topics in Software Defined Networking, HotSDN '14, ACM, New York, NY, USA, 2014, pp. 1-6. doi:10.1145/2620728.2620744. 


\section{Biography}

Guan $\mathrm{Xu}$ received the B.S. degree in Electronics and Information Engineering from Huazhong University of science and technology, Wuhan, P. R. China, in 2008. He is currently a PhD Candidate in the School of Electronic Information and Communications at the Huazhong University of science and technology. His research interests are in the areas of practical network coding in P2P network, IP switch networks and SDN networks with emphasis on routing algorithms and rate control algorithms.

Bin Dai received the B. Eng, the M. Eng degrees and the PhD degree from Huazhong University of Science and Technology of China, P. R. China in 2000, 2002 and 2006, respectively. From 2007 to 2009, he was a Research Fellow at the City University of Hong Kong. He is currently an associate professor at School of Electronic Information and Communications, Huazhong University of Science and Technology, P. R. China. His research interests include SDN network, wireless network, network coding.

Benxiong Huang received the B.S. in 1987 and Ph.D. in 2003 from HUST. He is currently a professor at School of Electronic Information and Communications, Huazhong University of Science and Technology, P. R. China. His research interests include signal processing and network security.

Jun Yang received the PhD degree from Huazhong University of Science and Technology of China, P. R. China in 2013. He is currently a Postdoctoral Fellow in Huazhong University of Science and Technology. His research interests include network coding and software-defined network.

Sheng Wen received the PhD degree from Deakin University and Central South University. He worked with Prof. Ivan Stojmenovic as Research Fellow in Deakin University, Australia in 2014. He is currently a lecturer in computer science in Deakin University. His research interests include modeling of the propagation and defense study of Internet malicious information. He is also interested in the techniques of identifying information origins in networks. 


\section{Biography}

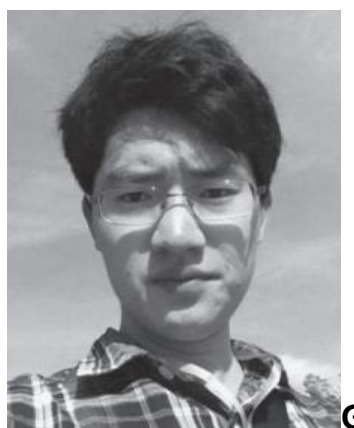

from Huazhong University of science and technology, Wuhan, P. R. China, in 2008. He is currently a $\mathrm{PhD}$ Candidate in the School of Electronic Information and Communications at the Huazhong University of science and technology. His research interests are in the areas of practical network coding in P2P network, IP switch networks and SDN networks with emphasis on routing algorithms and rate control algorithms.

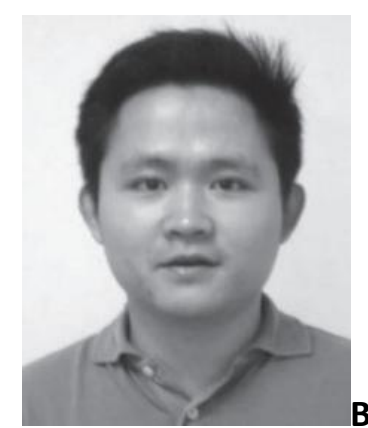

Bin Dai received the B. Eng, the $\mathrm{M}$. Eng degrees and the $\mathrm{PhD}$ degree from Huazhong University of Science and Technology of China, P. R. China in 2000, 2002 and 2006, respectively. From 2007 to 2009, he was a Research Fellow at the City University of Hong Kong. He is currently an associate professor at School of Electronic Information and Communications, Huazhong University of Science and Technology, P. R. China. His research interests include SDN network, wireless network, network coding.

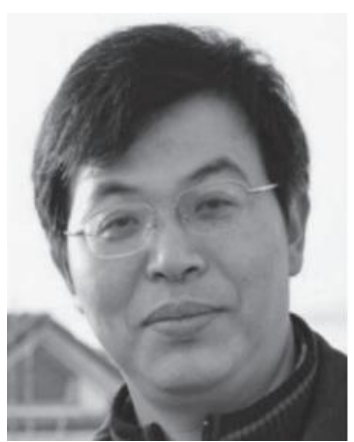

Benxiong Huang received the B.S. in 1987 and Ph.D. in 2003 from HUST. He is currently a professor at School of Electronic Information and Communications, Huazhong University of Science and Technology, P. R. China. His research interests include signal processing and network security. 


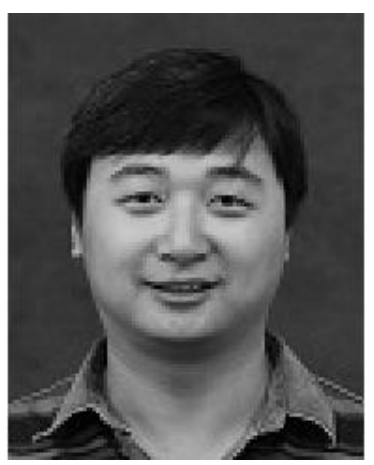

Jun Yang received the PhD degree from Huazhong University of Science and Technology of China, P. R. China in 2013. He is currently a Postdoctoral Fellow in Huazhong University of Science and Technology. His research interests include network coding and softwaredefined network.

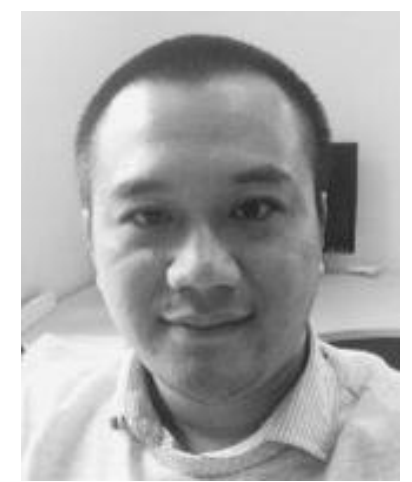

Sheng Wen received the PhD degree from Deakin University and Central South University. He worked with Prof. Ivan Stojmenovic as Research Fellow in Deakin University, Australia in 2014. He is currently a lecturer in computer science in Deakin University. His research interests include modeling of the propagation and defense study of Internet malicious information. He is also interested in the techniques of identifying information origins in networks. 